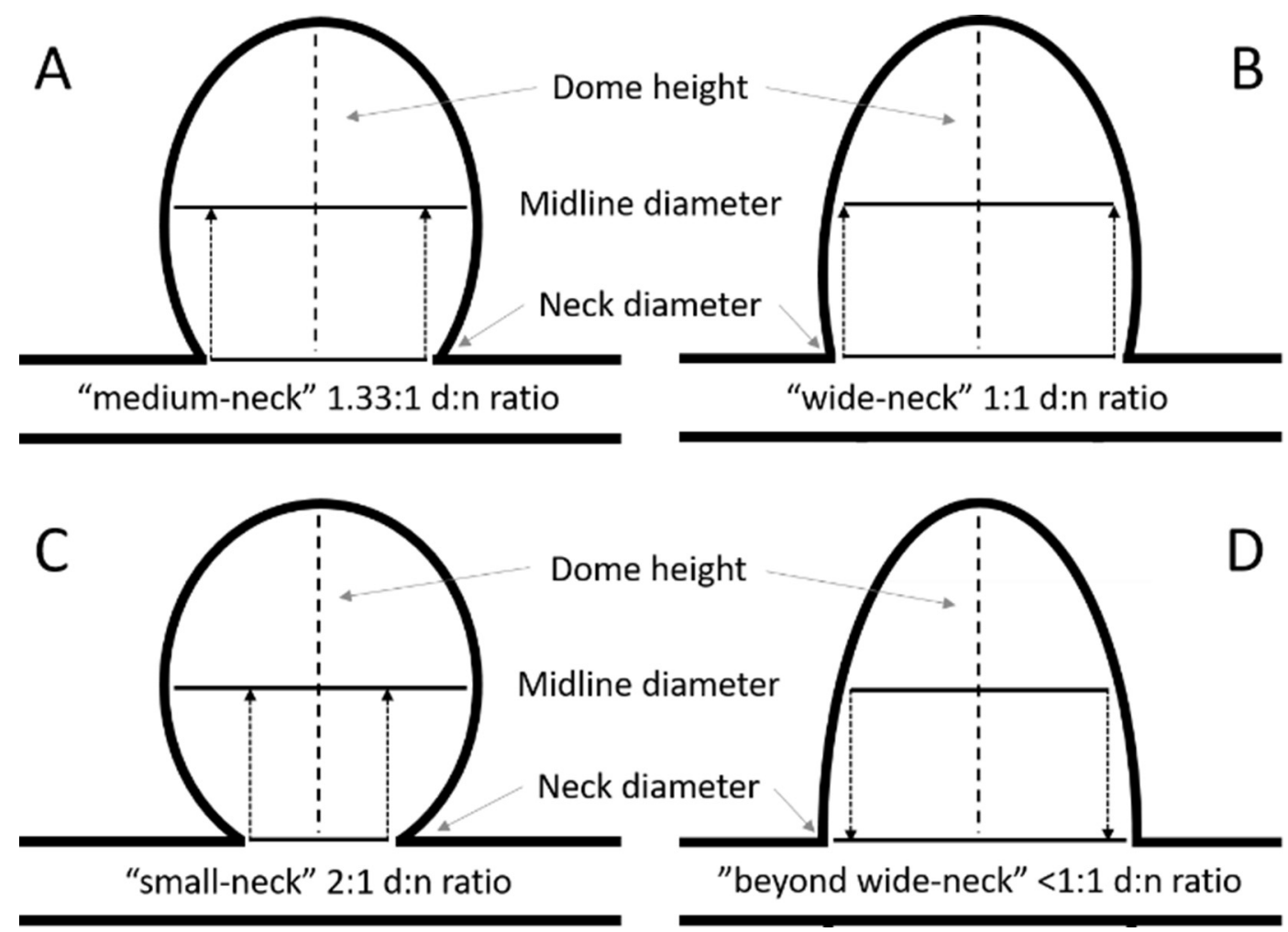

Abstract E-051 Figure 1 An illustration depicting the different d:n ratio classifications that may be possible. From the illustration, it can be inferred how certain devices may perform poorly in certain dome to neck diameter ratio classifications.

classification systems that practitioners, developers, and regulators may apply to intracranial aneurysms.

Disclosures W. Merritt: 1; C; NIH, Northern Arizona University. T. Becker: 1; C; NIH, Northern Arizona University. A. Ducruet: 1; C; NIH, Northern Arizona University. 5; C; Barrow Neurological Institute.

\section{E-052 ARTIFICIAL NEURAL NETWORK CT PERFUSION PREDICTION OF ISCHEMIC CORE}

${ }^{1} \mathrm{~A}$ Kasasbeh*, ${ }^{2} \mathrm{~S}$ Christensen, ${ }^{2} \mathrm{M}$ Lansberg. ${ }^{1}$ Radiology, University of Vermont, Colchester, $V T ;{ }^{2}$ Neurology, Stanford Stroke Center, Palo Alto, CA

\subsection{6/neurintsurg-2019-SNIS.127}

Background and purpose Computer Tomography Perfusion (CTP) is a useful tool in the evaluation of acute ischemic stroke, where it can provide an estimate of the ischemic core and the ischemic penumbra. The optimal CTP parameters to identify the ischemic core remain undetermined.

Methods We utilized Artificial Neural Networks (ANNs) to optimally predict the ischemic core in acute stroke patients, using diffusion-weighted imaging as the gold standard. We first designed an ANN based on CTP data alone and next designed an ANN based on clinical and CTP data.

Results The ANN based on CTP data predicted the ischemic core with a mean absolute error of $13.8 \mathrm{ml}$ (SD $13.6 \mathrm{ml}$ ) compared to DWI. The area under the receiver operator characteristic curve (AUC) was 0.85. At the optimal threshold, the sensitivity for predicting the ischemic core was 0.90 and the specificity was 0.62. Combining CTP data with clinical data available at time of presentation resulted in the same mean absolute error $(13.8 \mathrm{ml})$ but lower SD $(12.4 \mathrm{ml})$. Furthermore, the AUC, sensitivity, and specificity were $0.87,0.91$, and 0.65 , respectively. The maximal Dice coefficient was 0.48 in the ANN based on CTP data exclusively.

Conclusions An artificial neural network that integrates clinical and CTP data predicts the ischemic core with accuracy.

Disclosures A. Kasasbeh: None. S. Christensen: 2; C; Dr. Søren Christensen is an equity shareholders in iSchemaView and perform consulting work for iSchemaView. 4; C; Dr. Søren Christensen is an equity shareholders in iSchemaView and perform consulting work for iSchemaView. M. Lansberg: None.

\section{E-053 RADIAL ARTERY CATHETERIZATION FOR NEUROENDOVASCULAR PROCEDURES: CLINICAL OUTCOMES AND PATIENT SATISFACTION MEASURES}

0 Khanna*. Department of Neurological Surgery, Thomas Jefferson University Hospital, Philadelphia, PA

\subsection{6/neurintsurg-2019-SNIS.128}

Background and purpose The majority of neuroendovascular procedures are still performed via transfemoral access. Radial artery catheterization is an alternate route of access that has started to gain more widespread use for neuroendovascular procedures, and there have been few studies that describe its safety and efficacy. We present our institution's experience in performing neuroendovascular interventions via a transradial approach, with excellent clinical outcomes and patient satisfaction measures.

Methods We conducted a retrospective analysis and identified 223 patients who underwent 233 consecutive neuroendovascular interventions via radial artery access at our institution. The 